\title{
The lag time in initiating clinical testing of new drugs in combination with radiation therapy, a significant barrier to progress?
}

\author{
P Blumenfeld ${ }^{1,2}$, R M Pfeffer ${ }^{2,3,4}$, Z Symon ${ }^{2,3,4}$, R B Den $^{5}$, A P Dicker ${ }^{5}$, D Raben ${ }^{6}$ and Y R Lawrence ${ }^{\star, 3,4,5}$ \\ ${ }^{1}$ Department of Radiation Oncology, Rush University Medical Center, Chicago, IL, USA; ${ }^{2}$ Sackler School of Medicine, Tel Aviv \\ University, Tel Aviv, Israel; ${ }^{3}$ Department of Radiation Oncology, Sheba Medical Center, Ramat Gan, Israel; ${ }^{4}$ MSR School of \\ Radiotherapy, Ramat Gan, Israel; ${ }^{5}$ Department of Radiation Oncology, Sidney Kimmel Medical College at Thomas Jefferson \\ University, Philadelphia, PA, USA and ${ }^{6}$ Department of Radiation Oncology, University of Colorado Denver, Denver, CO, USA
}

Background: The clinical development of new drugs with radiation appears to be limited. We hypothesised that phase I clinical trials with radiation therapy (RT) are initiated too late into a new drug's lifetime, impeding the ability to complete RT-drug development programmes before patent expiration.

Methods: We identified novel drug-radiation phase I combination trials performed between 1980 and 2012 within the PubMed and ClinicalTrials.gov databases. Data gathered for each drug included: date the initial phase I trial with/without RT was opened/ published, date of the published positive phase III trials, and patent expiration dates. Lag time was defined as the interval between opening of the phase I trial without RT and the opening of the phase I with RT. Linear regression was used to model how the lag time has changed over time.

Results: The median lag time was 6 years. The initial phase I trial with RT was typically published 2 years after the first published positive phase III trial and 11 years before patent expiration. Using a best-fit linear model, lag time decreased from 10 years for phase I trials published in 1990 to 5 years in 2005 (slope significantly non-zero, $P<0.001$ ).

Conclusions: Clinical drug development with RT commences late in the life cycle of anti-cancer agents. Taking into account the additional time required for late-phase clinical trials, the delay in initiating clinical testing of drug-RT combinations discourages drug companies from further pursuing RT-based development. Encouragingly, lag time appears to be decreasing. Further reduction in lag time may accelerate RT-based drug development, potentially improving patient outcomes.

The addition of concomitant chemotherapy to radiation therapy (RT) has increased the cure rate for many types of cancer, and quantitatively is one of the most important advances in cancer care over the past 30 years (Seiwert et al, 2007). In parallel, advances in our understanding of cancer biology over the past two decades have led to the development of a new generation of targeted treatments (Lawrence et al, 2013b). Although there is good rationale and solid preclinical data supporting the utility of combining many of these new agents with RT, the clinical development of new radiation-drug combinations appears to be limited (Lawrence et al, 2013a). Very few of these newer biological agents have been combined with RT in phase III trials, and only one (cetuximab) has received a specific FDA-approved indication (Bonner et al, 2006).

We hypothesised that, one reason for this lack of progress may be that phase I clinical trials with RT are initiated late into a new drug's lifetime (i.e., close to the patent-expiry date). We investigated the relationship between the initial clinical testing of a new drug as a single-agent, initial clinical testing with radiation, and patent-expiry dates. 


\section{MATERIALS AND METHODS}

A search was performed to identify phase I trials involving novel drug-radiation combinations within the PubMed and ClinicalTrials.gov databases. For PubMed the search terms used were: (phase I) AND (radiation OR radiotherapy OR chemoradiotherapy OR chemoradiation). Specific limits that were placed on the PubMed search were Publication Dates 1980-2012; Species Humans; Language English; and Article Type Clinical Trials. For ClinicalTrials.gov an advanced search was performed using the terms (radiation OR radiotherapy OR chemoradiotherapy OR chemoradiation) and selecting phase I as an additional criterion.

Exclusion criteria were: (1) if the year that the clinical trial combining drug and radiation opened could not be determined from either the PubMed abstract, the complete manuscript, or the ClinicalTrails.gov website; (2) drugs that were pure radiosensitisers (i.e., drugs that were not tested as single agents in the absence of RT); and (3) trials of non-malignant pathologies.

For each drug for which a phase I trial involving concomitant radiation could be identified, we went back to identify additional time points that are as follows:

1. The opening of the initial phase I trial without RT by searching the PubMed and ClinicalTrials.gov databases.

2. The publication date of the initial phase I trial without RT.

3. The year of publication of the first positive phase III study of the drug without RT, as determined from the PubMed database. A positive study was defined as one that showed a significant difference $(P<0.05)$ in the primary end point (i.e., overall survival, disease-free survival, and cause-specific survival) of the study group.

4. Drug type categorised as chemotherapeutic, antibody, or targeted agent.

5. Sponsorship of the trial categorised as academic, industry, combined, or not-stated based on the information provided on the Clinicaltrials.gov database.

6. Initial FDA approval.

7. Patent expiration as determined from DrugPatentWatch (Friedman, 2012) and other websites.

Two definitions of lag time were pragmatically defined to account for missing data, especially for older drugs. Lag time-O was defined as the interval from the opening of the phase I trial without radiation until the opening of a phase I trial with radiation. Lag time-P was defined as the interval from publication of phase I trial without radiation until the opening of a phase I trial with radiation.

Statistical analysis. Statistical analyses were performed using the Stata Statistical package (version IC 11.1, StataCorp LP, College Station, TX, USA). Univariate logistic regression was used to assess variables that were associated with a longer lag time. A $P$-value of $<0.05$ was considered statistically significant. Linear regression was used to model how lag time has changed over time. Graphs were created using Prism 6 (GraphPad Software, Inc., La Jolla, CA, USA).

\section{RESULTS}

Eighty-four drugs were identified for which an initial phase I trial involving concomitant radiation could be obtained from the Pubmed and ClinicalTrials.gov databases. Of these, 18 drugs were excluded (for 13, the date the RT trial opened was not stated and for 5 , the drug had not been tested in the absence of radiation), see Figure 1 . The remaining 66 drugs formed the study population, consisting of 11 monoclonal antibodies, 20 chemotherapeutic cytotoxic agents, and 35 targeted small molecules. Sponsorship for the phase I trials was identified for 66 of the trials with RT and 58 of the trials without RT. Of those trials with RT, $68 \%$ of the sponsorship was academic, $23 \%$ was from industry, and $9 \%$ was combined; whereas for the trials without RT, $48 \%$ was academic, $39 \%$ was from industry, and $12 \%$ not stated (Supplementary Table S1). Non-radiation trials were more likely to be sponsored by the industry than radiation phase I trials (39\% vs 23\%), $(P$-value $<0.01)$.

Not all time points were obtainable for all drugs (Table 1); therefore, lag time-O could be calculated for 47 drugs and lag time$\mathrm{P}$ for 65 drugs. The median lag time-O between the opening of the phase I trial without RT and the opening of the phase I with RT was 6 years (interquartile range $5-8$ years). The median lag time-P between the published phase I trial without RT and the opening phase I with RT was 3 years (interquartile range 1-6 years), see Table 2. On the basis of these median time points, we created a typical timeline for the clinical development of a novel anti-cancer agent with radiation (Figure 2). Further analysis was based on the lag time-P variable as this was available for all 66 drugs.

Lag time-P was associated with the year of publication of the initial phase I trial without RT on linear regression $(P<0.001)$. Neither drug-class nor sponsorship of the RT phase I trial were associated with lag time (Table 3). Using a best-fit linear model, the lag time-P was shown to have decreased from 9 years for phase I trials published in 1990 to 3 years for trials published in 2005 (slope significantly non-zero, $P<0.001$ ), see Figure 3. Likewise, lag time-O was found to have decreased from 10 years in 1990 to 5 years in 2005 (slope significantly non-zero, $P<0.001$ ), (Supplementary Figure 1). The website ClinicalTrials.gov was created in 2000, and the International Committee of Medical Journal Editors (ICMJE) began to require trial registration as a condition of publication only in 2005. We were concerned that the decrease in lag time seen in recent years may be the result of bias resulting from the creation of the database. We therefore performed an additional analysis confined to dates obtainable from the manuscripts themselves without reference to ClinicalTrials.gov. For this analysis, we looked at the lag time from publication of phase I without RT to publication of phase I with RT (the opening dates were very rarely listed in the manuscript) for 44 drugs for which dates were available. Similarly, this analysis showed the time interval decreased from 12 years for RT trials published in 1990 to 5 years for RT trials published in 2005 (Supplementary Figure 2).

\section{DISCUSSION}

We have demonstrated that clinical drug development with RT commences late in the life cycle of anti-cancer agents, a median 6 years after the opening of the first phase I trial without RT and 15 years before patent expiration. Positively, we discovered that lag times have decreased in recent years, although they remain long.

To our knowledge, this is the first study to have quantified the lag time in initiating clinical trials with RT for novel anti-cancer agents. Our finding of a decrease in lag time in recent years is encouraging, although the reasons behind this shift are not evident. Ataman et al (2012) recently noted that combination chemo-RT trials using novel agents were not initiated until after drug approval, suggesting the existence of 'lag between the proof of clinical activity of novel targeted agents and the initiation of clinical trials of their use in combination with radiation therapy.' Our work, however, shows that phase I trials are initiated on an average 1 year before FDA approval. This discrepancy may be explained by the difference in methodology: Atamen's study analysed specifically targeted agents and looked at all phase I trials, 
PubMed search: (phase I) And (radiation OR radiotherapy OR chemoradiation OR chemoradiotherapy);

Limits: '1980-2012'; 'Clinical trial, phase 1'; 'humans'; 'english'
ClinicalTrials.Gov search: (radiation OR radiotherapy OR chemoradiation OR chemoradiotherapy);

Additional critera: 'phase 1'

Figure 1. Flow diagram of the process used to Identify trials for analysis.

\begin{tabular}{|l|c|c|c|}
\hline \multicolumn{3}{|c|}{ Table 1. Time points obtained for the analysed drugs and trials } \\
\hline & $\begin{array}{c}\text { Number } \\
\text { of drugs } \\
\text { for which } \\
\text { data } \\
\text { obtainable }\end{array}$ & Median & Range \\
\hline Time points & 47 & 2001 & $1960-2008$ \\
\hline Year of first trial without RT opened & 65 & 2002 & $1963-2011$ \\
\hline Year of first trial without RT published & 66 & 2005 & $1983-2012$ \\
\hline Year of first trial with RT opened & 45 & 2006 & $1996-2012$ \\
\hline Year of first trial with RT published & 45 & 2003 & $1979-2012$ \\
\hline Year of first phase III published & 34 & 1999 & $1967-2011$ \\
\hline Year of FDA approval & 34 & 2014 & $1994-2021$ \\
\hline Year of patent expiry & $\begin{array}{c}|c| \\
\text { Abbreviations: FDA = Food and Drug Administration; RT=radiation therapy. }\end{array}$ \\
\hline
\end{tabular}

categorising them by the location of tumour site, whereas our study looked at the first combination study for the specific drug and was not site specific.

Our manuscript has a number of limitations, and the potential sources of bias are: (1) the small number of new drugs analysed; however, this is an inevitable reflection of the limited number of trials performed with RT. (2) The ClinicalTrials.gov registry was created in 2000, and the ICMJE began to require trial registration as a condition of publication only in 2005; as the opening date listed in ClinicalTrials.gov may precede the true opening date, this may create a false impression that lag time has decreased in recent years. In order to overcome this, we performed an additional analysis, which ignored ClinicalTrials.gov and was based entirely on manuscript publication dates. This analysis similarly demonstrated a decrease in lag time from 12 to 5 years. (3) Not all trials listed in ClinicalTrials.gov are truly active-some may never be completed or even accrue patients. (4) For each drug, we searched for the first published trial without/with radiation. Unfortunately, not all completed trials are published (publication bias) (Dickersin et al,

\begin{tabular}{|c|c|c|}
\hline & \multicolumn{2}{|c|}{$\begin{array}{l}\text { Time-interval from } \\
\text { publication date of initial } \\
\text { phase I trial without } \\
\text { radiation }\end{array}$} \\
\hline Time points & Median & $\begin{array}{c}\text { Interquartile } \\
\text { range }\end{array}$ \\
\hline Opening of first trial without RT & -3 & 4 to 2 \\
\hline Opening of first trial with RT & 3 & 1 to 6 \\
\hline Publication of first trial with RT published & 7 & 5.5 to 12 \\
\hline Publication of first phase III published & 5 & 3 to 9 \\
\hline FDA approval & 4 & 1 to 6 \\
\hline Patent expiry & 18 & 13 to 20 \\
\hline
\end{tabular}

1987). We did not consider trials that were only published in the abstract form as the information contained therein was insufficient (e.g., they rarely mention as to when was the trial opened).

Much clinical research is funded by the pharmaceutical industry (Rettig, 2000), even within the NCI cooperative group network. Consequently, the majority of drug development occurs within a specific time window that terminates a number of years before patent expiration (Serajuddin and Serajuddin, 2006). Our study suggests a relative lack of interest by drug companies to perform clinical trials with RT, as demonstrated by the fact that phase I trials without RT were much more likely to be sponsored by the industry than phase I trials with RT. The reasons for the lack of interest of the drug industry are complex but may include an (unjustified?) fear of radiation-associated toxicity, and their reluctance to invest resources in an unproven drug. Taking into account the additional time required for late-phase (i.e., phase II/III) 


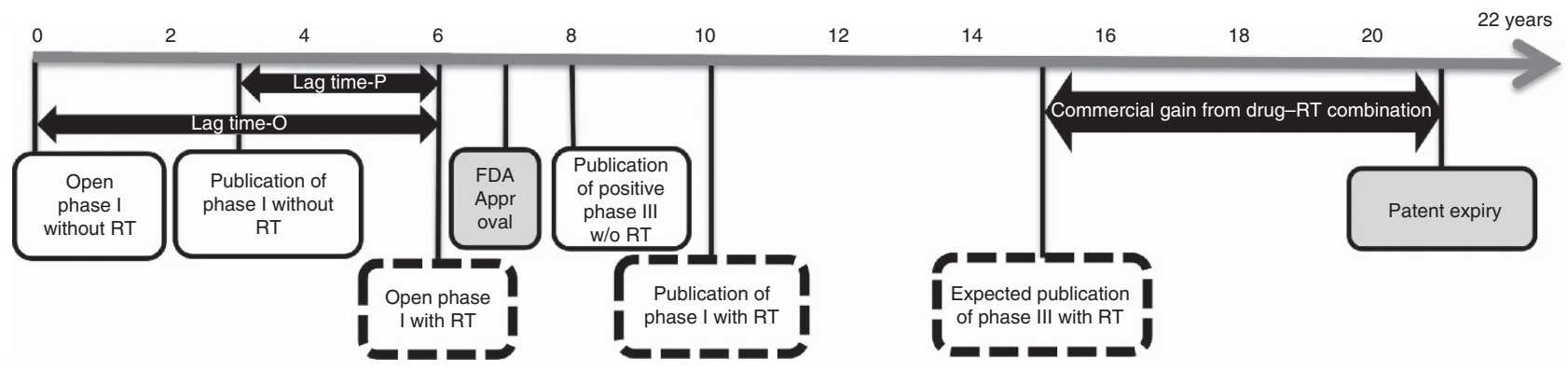

Figure 2. Typical time line of drug development with radiation. Time intervals refer to median values derived from the study of 66 drugs, as detailed in Table 2.

\begin{tabular}{|c|c|c|}
\hline Variable & Coefficient & $P$-value \\
\hline Year published without RT & 0.523 & $<0.001$ \\
\hline Drug class & -1.528 & 0.106 \\
\hline Sponsorship of RT trial & -1.738 & 0.111 \\
\hline
\end{tabular}

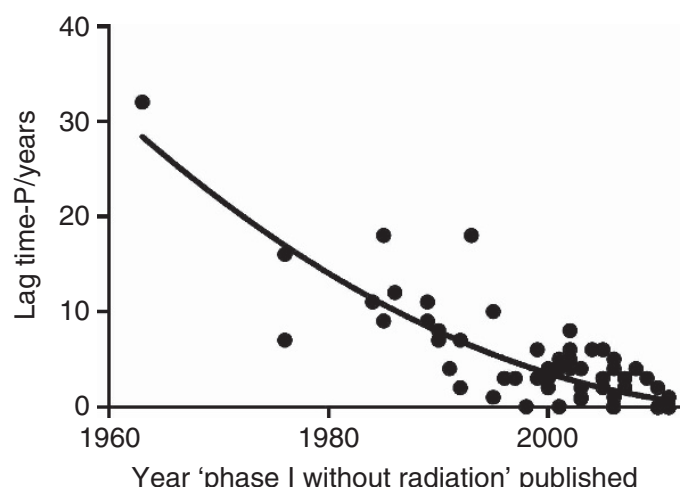

Figure 3. Lag time-P as a function of the publication year of phase I without RT.

clinical trials, this delay in initiating clinical testing of drug-RT combinations produces an additional disincentive for drug companies to pursue drug development with RT, as it becomes nearly impossible to obtain new, approved indications before patent expiration.

Lack of interest by the pharmaceutical industry in drug development with RT has been noted in the literature; with one reason being that the pharmaceutical industry does not view radiation combination trials as a path to drug registration (Lawrence et al, 2013b; Lin et al, 2013). Barton and Emmanuel (2005) have discussed the complex role that patent expiry has in drug development. On the one hand patent expiry is an incentive to innovation (Magazzini et al, 2004), on the other hand it places a time limit on a company's interest in any particular agent. Hence, the issues highlighted in this paper are a reflection of the bigger issues that market demand rather than genuine health needs often determine research priorities (Barton and Emanuel, 2005; Viergever, 2013). The proposed solutions include increased public-private cooperation and increased governmental funding of the areas that the industry neglects (i.e., radiation-based drug development) (Viergever, 2013).
There are likely other reasons for the observed delay in performing phase I trials with RT. To some extent this is an issue of scale- there are far more medical oncologists than radiation oncologists, e.g. in the USA there are 14000 medical oncologists but only 4000 radiation oncologists (Smith et al, 2010; Kirkwood et al, 2013). Within the specialty, there is also a lack of suitably trained personnel: developing phase I trials requires solid preclinical work in the lab, which is then followed up in a clinical trial. Unfortunately, the number of trained radiation biologists appears to be dropping (Rosenstein et al, 2009). Also, there are comparatively few physicians who have the skill sets and appropriate institutional support network to perform phase I studies with radiation. Phase I trials without radiation are generally performed in highly specialized and experienced high-throughput units. Conversely, radiation phase I trials appear to be performed by individual academic investigators working within busy clinical departments.

Drug-radiation trials are by definition multi-modality and hence logistically complex, creating a number of unique issues that can be sources of disagreement with, for example, scientific and ethical review boards (Lawrence et al, 2013b): How should the drug and radiation treatments be coordinated? How is toxicity attributed? Which modality should be adjusted when side effects occur? How should dose escalation be performed and what is the appropriate dose of drug (not necessarily the maximal tolerated dose)? A further issue is that the patient populations for non-RT and RT phase I trials are quite distinct. Whereas most non-RT phase I trials are performed in the advanced metastatic setting, for patients lacking additional standard options, radiation phase I trials often involve adding novel agents to the first-line therapy, attempting to cure the patient of locally advanced cancer.

While choosing which agent to combine with radiation, there is a recent trend to concentrate upon new pharmaceutical agents that have demonstrated activity as mono-agents (Serajuddin and Serajuddin, 2006). However, it is quite conceivable that a drug may enhance radiation-cell kill without having noticeable monotherapy activity (e.g., a pure DNA repair inhibitor). A new opportunity for drug development with radiation is provided by the emerging class of immunomodulating agents, for example, programmed death-1 (PD-1) inhibitors that are generating much interest as anti-cancer agents in melanoma and other solid tissues. There is evidence that radiotherapy/radiosurgery can uncover cancer cell antigens, stimulate the immune response and potentiate response to these agents (Formenti and Demaria, 2013; Lawrence and Dicker, 2014).

Although the lag time has decreased considerably over the last few years, there is a need to further shorten lag times to promote the viability of RT-drug combination development programmes. Increased cooperation between industry and academic radiation oncologists is required, to this end, both a European group and NCI/RTOG recently published guidelines to encourage a more streamlined approach for drug development with radiation 
(Harrington et al, 2011; Lawrence et al, 2013b). In addition to better collaboration between pharmaceutical companies and radiation oncologists, incentivising pharmaceutical companies to get more involved in radiation studies, improved trial designs, and guidance from the regulatory authorities to determine acceptable end points for radiation studies are imperative to successfully improving lag time. Increased non-industry funding for radiationbased trials would help. Alternatively, patent expiry dates could be extended, although this would be complicated.

A number of other variables may potentially be associated with lag time and will be the subject of future studies, for example, the severity of toxicity when the drug is used alone, the extent of preclinical data with RT, accrual rate for studies, and experience of combining similar sister drugs with radiation.

In conclusion, we have quantified the lag time in the clinical testing of new drugs with radiation. We have shown that there is a trend of decreasing lag times in recent years, although they remain too long. Although the combination of radiation with novel agents holds much promise for improving cancer cure rates, drug development with radiation is often initiated too late into a drug's patent lifetime to make the process commercially viable. Shortening the lag time will promote the commercial viability of developing novel radiation-drug combinations.

\section{REFERENCES}

Ataman OU, Sambrook SJ, Wilks C, Lloyd A, Taylor AE, Wedge SR (2012) The clinical development of molecularly targeted agents in combination with radiation therapy: a pharmaceutical perspective. Int J Radiat Oncol Biol Phys 84(4): e447-e454.

Barton JH, Emanuel EJ (2005) The patents-based pharmaceutical development process: rationale, problems, and potential reforms. JAMA 294(16): 2075-2082.

Bonner JA, Harari PM, Giralt J, Azarnia N, Shin DM, Cohen RB, Jones CU, Sur R, Raben D, Jassem J, Ove R, Kies MS, Baselga J, Youssoufian H, Amellal N, Rowinsky EK, Ang KK (2006) Radiotherapy plus cetuximab for squamouscell carcinoma of the head and neck. N Engl J Med 354(6): 567-578.

Dickersin K, Chan S, Chalmers TC, Sacks HS, Smith H Jr (1987) Publication bias and clinical trials. Control Clin Trials 8(4): 343-353.

Formenti SC, Demaria S (2013) Combining radiotherapy and cancer immunotherapy: a paradigm shift. J Natl Cancer Inst 105(4): 256-265.

Friedman Y (2012) DrugPatentWatch http://www.drugpatentwatch.com.

Harrington KJ, Billingham LJ, Brunner TB, Burnet NG, Chan CS, Hoskin P, Mackay RI, Maughan TS, Macdougall J, McKenna WG, Nutting CM, Oliver A, Plummer R, Stratford IJ, Illidge T (2011) Guidelines for preclinical and early phase clinical assessment of novel radiosensitisers. Br J Cancer 105(5): 628-639.

Kirkwood MK, Kosty MP, Bajorin DF, Bruinooge SS, Goldstein MA (2013) Tracking the workforce: the American Society of Clinical Oncology workforce information system. J Oncol Pract 9(1): 3-8.

Lawrence YR, Dicker AP (2014) Radiation therapy and the immune system: learning to live together. Future Oncol 10(5): 777-780.

Lawrence YR, Glass C, Symon Z, Dicker AP, Den RB (2013a) Phase I trials involving radiation therapy, quantifying the risks. J Med Imaging Radiat Oncol 57(6): 719-724.

Lawrence YR, Vikram B, Dignam JJ, Chakravarti A, Machtay M, Freidlin B, Takebe N, Curran Jr. WJ, Bentzen SM, Okunieff P, Coleman CN, Dicker AP (2013b) NCI-RTOG translational program strategic guidelines for the early-stage development of radiosensitizers. J Natl Cancer Inst 105(1): 11-24.

Lin SH, George TJ, Ben-Josef E, Bradley J, Choe KS, Edelman MJ, Guha C, Krishnan S, Lawrence TS, Le QT, Lu B, Mehta M, Peereboom D, Sarkaria J, Seong J, Wang D, Welliver MX, Coleman CN, Vikram B, Yoo S, Chung CH. Participants on Workshop for Preclinical and Clinical Development of RadiosensitizersNational Cancer Institute (2013) Opportunities and challenges in the era of molecularly targeted agents and radiation therapy. J Natl Cancer Inst 105(10): 686-693.

Magazzini L, Pammolli F, Riccaboni M (2004) Dynamic competition in pharmaceuticals. Patent expiry, generic penetration, and industry structure. Eur J Health Econ 5(2): 175-182.

Rettig RA (2000) The industrialization of clinical research. Health Aff 19(2): $129-146$.

Rosenstein BS, Held KD, Rockwell S, Williams JP, Zeman EM (2009) American Society for Radiation Oncology (ASTRO) survey of radiation biology educators in U.S. and Canadian radiation oncology residency programs. Int J Radiat Oncol Biol Phys 75(3): 896-905.

Seiwert TY, Salama JK, Vokes EE (2007) The concurrent chemoradiation paradigm-general principles. Nat Clin Pract Oncol 4(2): 86-100.

Serajuddin HK, Serajuddin AT (2006) Value of pharmaceuticals: ensuring the future of research and development. J Am Pharm Assoc 46(4): 511-516.

Smith BD, Haffty BG, Wilson LD, Smith GL, Patel AN, Buchholz TA (2010) The future of radiation oncology in the United States from 2010 to 2020: will supply keep pace with demand? J Clin Oncol 28(35): 5160-5165.

Viergever RF (2013) The mismatch between the health research and development $(\mathrm{R} \& \mathrm{D})$ that is needed and the $\mathrm{R} \& \mathrm{D}$ that is undertaken: an overview of the problem, the causes, and solutions. Glob Health Action 6 : 22450.

This work is published under the standard license to publish agreement. After 12 months the work will become freely available and the license terms will switch to a Creative Commons AttributionNonCommercial-Share Alike 3.0 Unported License.

Supplementary Information accompanies this paper on British Journal of Cancer website (http://www.nature.com/bjc) 